\begin{tabular}{cccc}
\hline & $\begin{array}{c}\text { International Journal of Health Services } \\
\text { Research and Policy } \\
\text { www.dergipark.org.tr/ijhsrp }\end{array}$ \\
$\begin{array}{c}\text { INESEG } \\
\begin{array}{c}\text { ENGINATIONAL } \\
\text { SCIENCE ANG } \\
\text { EDUCATION GROUP }\end{array}\end{array}$ & e-ISSN: $2602-3482 \quad$ DOI: $10.33457 /$ ijhsrp.677859 & IJHSRP \\
\hline
\end{tabular}

Research Article

\title{
PREVALENCE AND RISK FACTORS FOR DIABETIC RETINOPATHY IN TURKEY: A SCREENING PROGRAMME USING NON MYDRIATIC CAMERA CONCISE TITLE: USING NON MYDRIATIC CAMERA FOR DIABETIC RETINOPATHY
}

\author{
Hakika ERDOĞAN*I (D) Nazmiye EROL ${ }^{2}$ (D) Nilgün YILDIRIM ${ }^{3}$ (D) \\ ${ }^{1}$ Maltepe University, Medicine Faculty Ophthalmology Department, Istanbul, Turkey \\ ${ }^{2}$ Osmangazi University, Medicine Faculty Ophthalmology Department, Eskisehir, Turkey \\ ${ }^{3}$ Osmangazi University, Medicine Faculty Ophthalmology Department, Eskisehir, Turkey \\ Corresponding author; dr.gercek@yahoo.com
}

\begin{abstract}
Early identification of patients at risk for diabetes mellitus (DM) and associated morbidities such as diabetic retinopathy $(D R)$ is essential for effective lifestyle intervention and treatment. Largescale, cost-effective, and minimally invasive screening programs are critical for this purpose. This study evaluated the prevalence and history-based risk factors for DR and whether the non-mydriatic fundus camera is a useful screening method in Turkey at local health centers. A total of 5182 adults 40 years and older were invited to local health centers in rural Eskisehir, Turkey, for DR risk factor assessment and screening by fundus examination. Fundal images were graded on-site by trained ophthalmology assistants for DR and diabetic macular edema (DME). Patients with DR and DME were referred to the tertiary center for follow-up. The severity of DR was associated with female sex, older age, longer duration of DM, insulin usage, lower body mass index (BMI), lower educational level, higher systolic and diastolic blood pressure, and poor control of blood glucose. DR was not associated with alcohol intake or smoking. DME was associated with DM duration and age. Identifying DR prevalence and risk factors is essential for disease control. Non-mydriatic fundus camera imaging proved useful for largescale DR screening.
\end{abstract}

Keywords: Diabet, diabetic retinopathy, non-mydriatic fundus camera, diabetic macular edema, screening program

Received: January 20, $2020 \quad$ Accepted: April 25, 2020

\section{Introduction}

It is projected that 592 million patients with DM will be living in worldwide in 2035 [1]. DR is the most common complication of the DM with microvascular damage. All patients with diabetes are at risk of developing DR [2]. 
DR classification into non-proliferative and proliferative DR. If the burden changes the macula, macular edema can occur [3] One-third of diabetic patients have DR[4] and account for $5 \%$ of all blindness [1].

Because of both economic damage and preventable cause of blindness, almost the working-aged group indicate the importance of early diagnosis and treatment of DR[3-5]. Regular eye examination and effective treatment are essential for DM and DR management [6].

Although it is known risk factors of DR, the mechanism and progression have not been fully understood, so prompt diagnosis is essential through efficient screening [7].

Approximately $50 \%$ percent of the diabetes patients are not diagnosed, or diagnosed patients are not follow up and regularly treated [8]. Therefore inexpensive, easily accessible, and highly diagnostic devices can be better for us to follow up.

The aim of the study to evaluate risk factors of DR in the area by non-mydriatic fundus camera and its efficacy for DR follow up.

\section{Materials Subjects and Methods}

This research was supported by the faculty with a research project named 'Evaluation of the relationship between intraocular pressure and demographics, ocular and systemic factors in the population over 40 years of age in Eskişehir area'. This study was designed by the glaucoma department of Osmangazi university medical faculty. For the screening program, those who live in that area were informed before the study begins. The participants were invited to family health centers. Five thousand one hundred eighty-one patients who applied for screening in health centers were examined. A questionnaire was first applied to the participants. Questionnaires consisted of two parts. As shown in the form, the first part of the questionnaire questioned demographic information. The second part was also interrogating DM.(tobacco and alcohol usage, education level, etc.)

The diagnosis of diabetes was made according to the patients' anamnesis and the medication they used. The photographs of the patients who answered the questions thoroughly were taken by the assistant of ophthalmology. When retinal photographs were taken, Kowa (Kowa, Nagoya, Japan) nonmydriatic fundus camera was used. Pupils were not dilated. AAO Preferred Practice scheme [9]was used for Diabetic Retinopathy monitoring and treatment. Participants who have DR and DME were directed to the tertiary care center. Patients did not undergo an additional examination.

\section{Ethical statement}

This is a cross-sectional study of all procedures conformed to the tenets of the Declaration of Helsinki, and the study was approved by the Osmangazi University institutional ethical review board. (Date:31.10.2006 //Approval number:2006-03).

\subsection{Inclusion criteria}

Over 40 years of age

Living in the Eskişehir province

Adapting to Fundus photography

\subsection{Exclusion criteria}

Patients whose retinal photographs could not be evaluated clearly. 
In the present study, fundus photographs of diabetic patients were scanned retrospectively. 415 photos of 643 DM patients could be evaluated clearly.

All procedures conformed to the tenets of the declaration of Helsinki, and the local ethics committee approved the study.

\subsection{Statistical Analysis}

Pearson chi-square, Mann Whitney U tests, Kruskal Wallis H test, One Way Analysis of Variance (ANOVA) was used. Kolmogorov-Smirnov and Shapiro-Wilk tests of normality were used to check the normality of variable distributions. The SPSS 22.0 program was used in all the analyses. A value of $\mathrm{p}<0.05$ was accepted as significant.

\section{Results}

A total of 5182 cases participated in the study were 3680 women (71\%) and 1501 men (29\%). Four hundred ninety-six women (9.5\%) and 147 men of the participants had diabetes (2.8\%). The rate of diabetes was $12.4 \%$ in the study. There was a statistically significant difference between male and female gender ( $\mathrm{p}<0.001)$. DM was seen more in women. The frequency of DR was found to be $21 \%$ in the participating diabetic community, while the frequency was found to be $2 \%$ when all participants were included. DR was found more frequently in the female gender $(p<0.05)$. The demographic data and risk factors of DR and DM was shown in Table 1.

Table 1.Characteristics of participants in the study

\begin{tabular}{|l|l|l|l|l|}
\hline & DM - & DR- & NPDR & PDR \\
\hline Age (years) & $55.43 \pm 0.15$ & $58.98 \pm 0.47$ & $57.96 \pm 1.1$ & $63.11 \pm 2.91$ \\
\hline $\begin{array}{l}\text { Gender } \\
\text { Male (n) }\end{array}$ & 1415 & 66 & & \\
Female (n) & 3351 & 262 & 17 & 3 \\
\hline BMI (kg/m ${ }^{2}$ ) & $28.16 \pm 0.4$ & $29.04 \pm 0.24$ & $28.87 \pm 0.7$ & $27.03 \pm 2.01$ \\
\hline Tabaco use (n) & 1022 & 50 & 4 & 2 \\
\hline Alcohol use (n) & 305 & 11 & 1 & 0 \\
\hline SBP (mmHg) & $139.69 \pm 1.46$ & $140.14 \pm 1.28$ & $143.17 \pm 4.36$ & $142.15 \pm 3.25$ \\
\hline DBP (mmHg) & $80.87 \pm 0.17$ & $82.72 \pm 0.6$ & $83.87 \pm 0.6$ & $82.69 \pm 2.15$ \\
\hline DME (n) & - & - & 17 & 2 \\
\hline Insulin usage & - & 15 & 7 & 3 \\
\hline BGLC & - & 292 & 53 & 9 \\
\hline
\end{tabular}

n, number of the participant; BMI, Body Mass Index; SBP, Systolic Blood Pressure (SBP); DPB, Diastolic Blood Pressure; DME, Diabetic Macular Edema; BCGL, Blood glucose control at every six months.

In the study, the prevalence of non-proliferative DR (NPDR) and proliferative DR (PDR) in DM patients, $16 \%$ and $5 \%$, respectively.

In the diabetic group, the mean age was found to be higher and statistically significant. $(\mathrm{p}<0.001)$. Although the mean age in the PDR group was higher than the NPDR group that was not statistically significant. 
Int. J. of Health Serv. Res. and Policy (2020) 5(1): 15-23

Participants' SBP and DBP values were as in the table. Both SBP and DBP were higher in both the diabetic patients and the patients had DR.

Participants were analyzed for BMI. BMI was higher in patients with diabetes and DR and it was statistically significant $(\mathrm{p}<0.05)$. But there was no difference between the patients with NPDR and PDR in terms of BMI ( $p>0.05)$.

The medication used by DM patients was investigated. Insulin usage increased as the severity of the DR increased. $(\mathrm{p}<0.05)$

In the present study, diabetic patients without DR measured blood glucose more frequently than the others $(\mathrm{p}<0.05)$.

The presence of DME in diabetic participants was investigated. Nineteen of diabetic patients had DME. 16 cases were women and 3 cases were men. There was a statistically significant difference between proliferative and non-proliferative groups in terms of the presence of DME ( $p$ <0.001). In the non-proliferative group, DME was higher.

The relationship between the duration of diabetes mellitus and DR was evaluated, as shown in table 2 . The incidence and severity of diabetic retinopathy were increasing as the duration of the disease increases $(\mathrm{p}<0.001)$.

Table 2. Duration of diabetes mellitus associated with diabetic retinopathy.

\begin{tabular}{|l|c|c|c|c|c|}
\hline & 5 years & $6-10$ years & $11-15$ years & $16-20$ years & 21 years \\
\hline Non-proliferative DR (n) & 11 & 27 & 10 & 4 & 4 \\
\hline Proliferative DR (n) & 1 & 1 & 1 & 3 & 3 \\
\hline Non-DR (n) & 149 & 113 & 37 & 16 & 12 \\
\hline
\end{tabular}

n: number of the participant; DR, Diabetic retinopathy.

Participants were assessed for diabetic retinopathy and education level, as shown in Table 3 . The education level was higher in the group which had not DR $(\mathrm{p}<0.05)$. However, there was no difference between the non-proliferative group and the proliferative group for education.

Table 3. Education level association with diabetic retinopathy.

\begin{tabular}{|l|l|l|l|l|}
\hline & Uneducated & Primary education & High school & University \\
\hline Nonproliferative DR (n) & 10 & 64 & 2 & 1 \\
\hline Proliferative DR (n) & 2 & 5 & 2 & - \\
\hline Non-DR (n) & 48 & 264 & 11 & 4 \\
\hline
\end{tabular}

$\mathrm{n}$, number of the participant; DR, Diabetic retinopathy.

\section{Discussion}

The prevalence of DR varies widely among geographic regions and demographic groups. Further, estimates within populations have differed markedly across studies. For instance, the rate of DR among DM patients was reported to be $9.6 \%$ in India10 but $43.1 \%$ in Indonesia.[11] In the present study, DR was identified in $21 \%$ of diabetic participants ( $2 \%$ of the entire participant cohort), an estimate consistent with one previous study of DR prevalence among DM patients in Turkey (23.6\%) but substantially lower than two others (36\% and 32.7\%) [12-14]. These differences among studies may be caused by 
insufficient sample size, leading to variation in risk factor distributions (e.g., age and disease duration) and (or) to the methods used for fundus examination [15].

In the current study, DR was significantly higher in diabetic females, in contrast to several previous studies reporting no significant sex difference in DR [16-19] or greater prevalence in males [4,20-23]. Participants were examined during working hours on weekdays, which may have increased the participation rate of women who do not work full time. Furthermore, most previous trials were hospital-based, so the frequency and severity of DR were higher. Also, the greater mortality of male diabetics may have influenced these hospital-based estimates. Thus, sampling bias may influence the relative prevalence of DR between sexes. Also, sex differences in lifestyle factors and genetic predisposition may contribute [21]. A wide-scale epidemiological study with careful sampling control is required to determine conclusively whether there is a female preponderance for DR among DM patients in Turkey and other regions.

Longer duration of diabetes and older age were associated with DR in the present study, consistent with several previous studies $[4,21,24,25]$. However, the presence of DR could not be attributed to participant age, as in some studies [21,24] even if diabetic patients were elderly [15,19]. Differences in other risk factors, such as body weight, blood pressure, and life span (i.e. earlier mortality in one population compared with another) may have obscured an effect of age alone.

Previous studies have reported an association between severe retinopathy and insulin treatment [19, 24, 26] by the current study. Also, using a hypoglycaemic agent was associated with DR [27]. Alternatively, a study in Hong Kong found a relatively high prevalence of microvascular complications despite a low insulin usage rate [28]. The years of initiation of insulin and the level at which the disease has started have not been explicitly stated in studies. This result suggests that the evaluation of only one factor associated with DR development may lead to a failure to follow disease progression.

Hypertension treatment has been associated with a higher incidence of DR [24], and high SBP, in particular, predicted a significantly greater incidence of DR $[4,15,18,19,21,25,27,29]$. These results were verified in the present study.

Although DME can be observed at any stage of DR, it is related to DR severity [30-32]. In the current study, however, DME was more common in patients with DR, but was not related to the severity of DR. This the result may be due to fewer proliferative DR participants, who are usually monitored more often.

Several studies have reported a direct relationship between DR and alcohol use [4, 27, 33], whereas others have not found such an association. [19,26,34,35] In one meta-analysis of studies, it was argued that some alcoholic beverage types protect against the development of retinopathy [5]. In the present study found no relationship between alcohol use and DR but did not record frequency and type of alcoholic beverages consumed and other dietary or social factors. This issue warrants further analysis [5]. The current study also evaluated the association between smoking and DR, and the absence of a relationship is in accord with several previous studies $[4,19,26]$ but the contrast to another [22]. The most important limiting factor in our study was that a large proportion of the participants were nonalcoholic and non-smoking females. Also, the amount of alcohol and the number of cigarettes consumed and how long they were used before stopping was not recorded. Glycaemic control is among the most important risk factors for DR $[2,3,7,21,36]$. In agreement with the current study, a previous investigation found that intensive glucose control reduced DR, although not all-cause mortality [37]. 
This study also revealed a significant relationship between low BMI and DR. Although there is strong evidence for an association of DR with BMI, the direction of the relationship has been inconsistent across studies, with several in accord with our findings[15,28,38] and others reporting a significant relationship between DR and high BMI [19,21,22,39]. These inconsistencies may stem from differences in the proportion of adipose tissue, diabetes severity, ketosis presence, and insulin secretion among study groups [1]. Finally, lower educational attainment was a significant risk factor for DR as in other studies $[19,28,40]$. Better communication on lifestyle choices and the importance of self-care (such as intensive glucose control) may improve DM management among this population.

Complications of diabetes, such as retinopathy, can be prevented by reducing lifestyle-related risk factors. Moreover, early diagnosis can increase the chances of slowing disease progression and successful treatment. Knowing the risk factors will help patients make appropriate choices and aid in the screening of high-risk populations. Regular screening is essential for these goals, so it is also important that the diagnostic methods are easily accessible, cost-effective, and minimally invasive to increase the reach of screening, such as a non-mydriatic fundus camera. These criteria are achieved using non-mydriatic fundus camera imaging.

Many of the aforementioned studies were hospitals based, and eyes were dilated during fundus imaging. Nonetheless, results were similar to the present study; when the information mentioned in this study is compared with the literature, there is a close resemblance, and these were done by evaluating only the photographs taken with a non-mydriatic fundus camera and patient information. Suggesting that the non-mydriatic fundus camera can also be used safely for screening and follow-up, especially in areas where access to a doctor is difficult and also non-mydriatic fundus camera with machine-learning programs will be able to reach wider audiences in the future.

We also had restrictive factors in our study. In the present study could not measure the participant's blood glucose levels, not use OCT and fundus fluorescein angiography in evaluating macular demand retinopathy and not evaluate vision

\section{Conclusion}

Complications of diabetic retinopathy is a health problem that can be detected and prevented. Knowing the risks of diabetic retinopathy, informing patients, and screening patients with these risks is important for survival. Patients with risk factors should be screened more often. The fact that most of the risk factors are changeable helps prevent the progression of the disease. So, it is also important that the screening methods are easy and accessible in terms of reaching many patients, such as non-mydriatic fundus cameras. In a short time, in the present study was screened a large group of participants with a non-invasive method. This study can be a pioneer for deep learning and machine learning-based programs. So, it can help an extensive health screening with less budget in the future. If the nonmydriatic fundus camera can be used with machine- learning, it seems that this will lead to significant public health.

\section{Conflict of interest}

The authors declare that they have no conflict of interest.

\section{Acknowledgments}

The research is a faculty supported individual research projects.

The data used to support the findings of this study may be provided upon request and the data should be submitted as an additional information file with email interaction. 
Int. J. of Health Serv. Res. and Policy (2020) 5(1): 15-23

Help for English editing was received from 'Enago' service.

The compliance to the Research and Publication Ethics: This study was carried out in acoordence with the rules of research and publication ethics.

\section{Ethical statement}

This is a cross-sectional study of all procedures conformed to the tenets of the Declaration of Helsinki, and the study was approved by the Osmangazi University institutional ethical review board. (Date:31.10.2006 //Approval number:2006-03).

\section{References}

[1] Chan, J.C.Y., et al.," Differential effect of body mass index on the incidence of diabetes and diabetic retinopathy in two Asian populations", Nutr Diabetes, 8(1), 16., 2018.

[2] Chatziralli, I.P., "The Role of Glycemic Control and Variability in Diabetic Retinopathy", Diabetes Ther, 9(1), 431-434, 2018.

[3] Kashim, R.M., P.," Newton, and O. Ojo, Diabetic Retinopathy Screening: A Systematic Review on Patients' Non-Attendance", International Journal of Environmental Research and Public Health, 15(1), 157, 2018.

[4] Thapa, R., et al., "Prevalence and risk factors of diabetic retinopathy among an elderly population with diabetes in Nepal: the Bhaktapur Retina Study", Clinical Ophthalmology (Auckland, N.Z.), 12, 561-568, 2018.

[5] Zhu, W., et al.," Association of alcohol intake with risk of diabetic retinopathy: a meta-analysis of observational studies", Sci Rep, 7(1), 4, 2017.

[6] Lingam, S., et al.," Knowledge, attitude and practices on diabetes, hypertension and diabetic retinopathy and the factors that motivate screening for diabetes and diabetic retinopathy in a pyramidal model of eye health care", Rural Remote Health, 18(1), 4304, 2018.

[7] Romero-Aroca, P., et al.," A Clinical Decision Support System for Diabetic Retinopathy Screening: Creating a Clinical Support Application", Telemed J E Health, 1; 25(1), 31-40,2019

[8] Nadarajan, B., et al., "Prevalence of Diabetic Retinopathy and its Associated Factors in a Rural Area of Villupuram District of Tamil Nadu, India", J Clin Diagn Res, 11(7), Lc23-lc26, 2017.

[9] Hall-Moller, J., K. Albrecht, and P. Lee, "Conformance with the preferred practice pattern for diabetic eye care", Retina, 18(2), 160-3, 1998.

[10] Jonas, J.B., et al., "Prevalence and Associated Factors of Diabetic Retinopathy in Rural Central India", Diabetes Care, 36(5), pp. e69-e69, 2013.

[11] Sasongko, M.B., et al., "Prevalence of Diabetic Retinopathy and Blindness in Indonesian Adults With Type 2 Diabetes", Am J Ophthalmol, 181, 79-87, 2017.

[12] Idil, A., Ocaktan E., "The prevalence of blindness and low vision in older onset diabetes mellitus and associated factors A community-based study", European Journal of Ophthalmology, 14(4), 298-305, 2004. 
Int. J. of Health Serv. Res. and Policy (2020) 5(1): 15-23

[13] Ulusoy, M,, Atakan, M.," Are Diabetic Patients Aware Of The Danger In Turkey?" Acta Medica Mediterranea, 32, 925, 2016.

[14] Tas, A., Erdem, U., "Diyabetik hastalarda retinopati gelisimine etki eden risk faktörlerinin degerlendirilmesi: çok merkezli çalısma (Türkiye'de Diyabetik Retinopati Epidemiyolojisi Arastrma Grubu)", Gülhane Tip Dergisi, 48, 94-100, 2006.

[15] Cui, J., et al., "Prevalence and associated factors of diabetic retinopathy in Beijing, China: a crosssectional study", BMJ Open, 7(8), p. e015473, 2017.

[16] Bertelsen, G., et al., "Tromso eye study: prevalence and risk factors of diabetic retinopathy", Acta Ophthalmol, 91(8), 716-21, 2013.

[17] Segato, T., et al., "The epidemiology and prevalence of diabetic retinopathy in the Veneto region of northeast Italy. Veneto Group for Diabetic Retinopathy", Diabet Med, 8, 11-6, 1991.

[18] Liu, Y., et al., "Risk factors of diabetic retinopathy and sight-threatening diabetic retinopathy: a cross-sectional study of 13473 patients with type 2 diabetes mellitus in mainland China", BMJ Open, 7(9), p.e016280, 2017.

[19] Xu, J., et al.," Ten-Year Cumulative Incidence of Diabetic Retinopathy. The Beijing Eye Study 2001/2011", PLoS ONE, 9(10), p. e111320, 2014.

[20] Zhang, X., et al., "Prevalence of diabetic retinopathy in the United States, 2005-2008", Jama, 304(6), 649-56, 2010.

[21] Liu, L., et al., "Incidence Density and Risk Factors of Diabetic Retinopathy Within Type 2 Diabetes: A Five-Year Cohort Study in China (Report 1)", International Journal of Environmental Research and Public Health, 12(7), 7899-7909, 2015.

[22] Tyrberg, M., et al.," Overweight, hyperglycemia, and tobacco use are modifiable risk factors for the onset of retinopathy 9 and 17years after the diagnosis of diabetes - A retrospective observational nation-wide cohort study", Diabetes Res Clin Pract, 133, 21-29, 2017.

[23] Sunita, M., et al., "Prevalence of Diabetic Retinopathy in Urban Slums: The Aditya Jyot Diabetic Retinopathy in Urban Mumbai Slums Study-Report 2", Ophthalmic Epidemiol, 24(5), 303-310, 2017.

[24] Jones, C.D., et al., "Incidence and Progression of Diabetic Retinopathy During 17 Years of a Population-Based Screening Program in England", Diabetes Care, 35(3), 592-6, 2012.

[25] Fredrick, T., et al., "Diabetic retinopathy and its risk factors in patients with type 2 diabetes attending rural primary healthcare facilities in Tamil Nadu", Natl Med J India, 29(1), 9-13, 2016.

[26] Yan, Z.P., J.X. Ma, "Risk factors for diabetic retinopathy in northern Chinese patients with type 2 diabetes mellitus", Int J Ophthalmol, 9(8), 1194-9, 2016.

[27] Martin-Merino, E., et al., "Risk factors for diabetic retinopathy in people with Type 2 diabetes: A case-control study in a UK primary care setting", Prim Care Diabetes, 10(4), 300-8, 2016.

[28] Kung, K., et al., "Prevalence of complications among Chinese diabetic patients in urban primary care clinics: a cross-sectional study", BMC Family Practice, 15, 8-8, 2014. 
Int. J. of Health Serv. Res. and Policy (2020) 5(1): 15-23

[29] Tan, G.S., et al., "Ethnic Differences in the Prevalence and Risk Factors of Diabetic Retinopathy: The Singapore Epidemiology of Eye Diseases Study", Ophthalmology, 125(4), 529-536, 2018.

[30] Klein, R., et al.," The Wisconsin epidemiologic study of diabetic retinopathy. XI. The incidence of macular edema", Ophthalmology, 96(10), 1501-10, 1989.

[31] Klein, R., et al.," The Wisconsin Epidemiologic Study of Diabetic Retinopathy XXIII: the twentyfive-year incidence of macular edema in persons with type 1 diabetes", Ophthalmology, 116(3), 497-503, 2009.

[32] Acan, D., et al.," The prevalence and systemic risk factors of diabetic macular edema: a crosssectional study from Turkey", BMC Ophthalmol, 18(1), 91, 2018.

[33] Young, R.J., et al., "Alcohol: another risk factor for diabetic retinopathy?", Br Med J (Clin Res Ed), 288(6423), 1035-7, 1984.

[34] Lee, C.C., et al.," Association between alcohol consumption and diabetic retinopathy and visual acuity-the AdRem Study", Diabet Med, 27(10), 1130-7, 2010.

[35] Moss, S.E., et al, "The association of alcohol consumption with the incidence and progression of diabetic retinopathy", Ophthalmology, 101(12), 1962-8, 1994.

[36] Billard, F., et al, Early worsening of diabetic retinopathy after rapid improvement of blood glucose control in patients with diabetes. Diabetes Metab, 44(1), 4-14, 2018.

[37] Hemmingsen, B., et al., "Intensive glycaemic control for patients with type 2 diabetes: a systematic review with meta-analysis and trial sequential analysis of randomised clinical trials", Bmj, 343, p. d6898, 2011.

[38] Rooney, D., et al., "Body mass index and retinopathy in Asian populations with diabetes mellitus", Acta Diabetol, 52(1), 73-80, 2015.

[39] Hammes, H.P., et al., "Risk Factors for Retinopathy and DME in Type 2 Diabetes-Results from the German/Austrian DPV Database", PLoS One, 10(7), p. e0132492, 2015.

[40] Emoto, N., et al., "A socioeconomic and behavioral survey of patients with difficult-to-control type 2 diabetes mellitus reveals an association between diabetic retinopathy and educational attainment", Patient Prefer Adherence, 10, 2151-2162, 2016. 